CLINICAL STUDY

\title{
Serum $\beta-2$ microglobulin predicts mortality in people with diabetes
}

\author{
Ching-Lung Cheung ${ }^{1}$, Karen S L Lam ${ }^{2,3}$ and Bernard M Y Cheung ${ }^{1,3}$ \\ ${ }^{1}$ Division of Clinical Pharmacology and Therapeutics, ${ }^{2}$ Division of Endocrinology, Department of Medicine and ${ }^{3}$ Research Centre of Heart, \\ Brain, Hormone and Healthy Aging, The University of Hong Kong, Pokfulam, Hong Kong, China \\ (Correspondence should be addressed to B M Y Cheung who is now at University Department of Medicine, Queen Mary Hospital, \\ 102 Pokfulam Road, Hong Kong, China; Email: mycheung@hku.hk)
}

\begin{abstract}
Objective: Serum $\beta-2$ microglobulin (B2M) level predicts mortality in chronic kidney disease. Glycation of $\mathrm{B} 2 \mathrm{M}$ is cytotoxic and may contribute to the risk of mortality in diabetic patients. Our objective was to evaluate the relationship between B2M and mortality in diabetic patients.

Methods: In this prospective study, 896 participants of the Third National Health and Nutrition Examination Survey with diabetes were included in the analysis. Serum B2M level was used in multivariate Cox regression analysis to predict all-cause and diabetes-related mortality.

Results: During a median follow-up of 11.8 years (range 0.1-18.2 years) and 9220.5 person/years, $541(42.4 \%)$ and $207(16.8 \%)$ participants died from all causes and diabetes-related causes respectively. One natural-log unit of $\mathrm{B} 2 \mathrm{M}$ was significantly associated with all-cause (hazard ratio $(\mathrm{HR})=6.53$, 95\% CI 2.07-20.6) and diabetes mortality (HR=7.35, 95\% CI 1.01-53.38) after multivariable adjustment. Similar results were obtained when B2M was analyzed as tertiles or in the threshold model (T1 + T2 vs T3). Examination of regression splines suggests a linear increase in hazard for mortality with increasing B2M levels.

Conclusions: Serum B2M level is a novel predictor of all-cause and diabetes-related mortality in people with diabetes regardless of renal function.
\end{abstract}

European Journal of Endocrinology 169 1-7

\section{Introduction}

Diabetes is one of the leading causes of death in the USA and the tools for predicting overall and cause-specific mortality in diabetic patients are limited. Chronic kidney disease (CKD) is a common complication of diabetes and is associated with cardiovascular disease (CVD) and increased mortality (1). Markers of CKD, such as albuminuria and low glomerular filtration rate (GFR), have been shown to predict mortality in people with diabetes (2).

$\beta$-2 Microglobulin (B2M) is a low molecular weight protein that is better than creatinine as a marker of renal function. An elevated serum level of B2M has been found to be associated with CVD $(3,4)$ and increased mortality $(3,4,5,6)$ in patients with renal dysfunction, in the elderly population as well as in the general population. Interestingly, although B2M is known to be a marker of renal function, the effect of $\mathrm{B} 2 \mathrm{M}$ on allcause mortality was found to be independent of renal function in the prospective study with 1034 nondisabled persons aged 65 years or older (6). B2M is susceptible to advanced glycation end product (AGE)modification and glycation. AGE-modification of B2M stimulates secretion of multiple pro-inflammatory cytokines, such as interleukin $1 \beta$ (IL1 $\beta$ ), IL6, and tumor necrosis factor $\alpha$ (TNF $\alpha)$, from human macrophages (7). In addition, glycation of $\mathrm{B} 2 \mathrm{M}$ renders it cytotoxic (8). Moreover, in two mouse models of type 1 diabetes, the expression of $\mathrm{B} 2 \mathrm{M}$ in retina was found to be significantly increased when compared with controls (9). These observations suggested that B2M is not only a marker of renal function but may also worsen outcome in diabetes. We hypothesize that B2M is a predictor of mortality in people with diabetes regardless of the degree of renal dysfunction.

\section{Research design and methods}

\section{Participant recruitment}

Data from the Third National Health and Nutrition Examination Survey (NHANES III) were used (10). The survey sample represented the civilian non-institutionalized USA population. Participants gave informed consent before participation, and ethical approval was obtained from the Human Subjects Committee of the USA Department of Health and Human Services. 
We included in our analysis diabetic participants aged more than 20 years whose serum B2M levels were available. Pregnant participants or participants with missing data in the multivariable models were excluded. After applying the exclusion criteria, 896 participants were included in the analysis (Supplementary Figure 1, see section on supplementary data given at the end of this article).

\section{Definition of diabetes}

Diabetes was defined as a fasting plasma glucose $\geq 126 \mathrm{mg} / \mathrm{dl}$ (fasted for $8-24 \mathrm{~h}$ ), an $\mathrm{HbAlc}$ value $\geq 6.5 \%$ self-reported diabetes, or self-reported current use of oral hypoglycemic medication or insulin.

\section{Independent variables}

Age, gender, race/ethnicity, smoking status, and physical activity were assessed using a questionnaire. In NHANES III, there were four race/ethnicity categories: white (non-Hispanic), black (non-Hispanic), Mexican-American, and 'other'. Education was categorized as less than high school, high school degree, or more than high school degree. We used the same definition for never, current, and former smokers that is used in the National Health Interview Surveys (NHIS) conducted by the Center for Disease Control (http://www.cdc.gov/nchs/nhis.htm). Individuals who had not smoked more than 100 cigarettes (pieces) in their lifetime were considered never smokers; those who had smoked more than 100 cigarettes in their lifetime were considered former smokers if they answered negatively to the question 'Do you smoke now?' and current smokers if they answered affirmatively. Participants who engaged in any physical activity with 3-5.9 metabolic equivalent tasks (METs) and 5 or more times per week or any physical activity with 6 or more METs and 3 or more times per week were classified as physically active (ideal category). BMI was calculated as weight in kilograms divided by height in meters squared. The estimated GFR (eGFR) was calculated from the CKD-EPI equation (11). Microalbuminuria was defined as albumin-creatinine ratio $\geq 30 \mathrm{mg} / \mathrm{g}$. Triglycerides $(\mathrm{mg} / \mathrm{dl})$, total cholesterol $(\mathrm{mg} / \mathrm{dl})$, HDL cholesterol $(\mathrm{mg} / \mathrm{dl})$, systolic blood pressure $(\mathrm{mm} / \mathrm{Hg})$, CRP $(\mathrm{mg} / \mathrm{dl})$, total white blood cell count (SI), and hemoglobin $(\mathrm{g} / \mathrm{dl})$ were also included as independent variables. Details regarding the measurement of the independent variables are available at the NHANES website (http://www.cdc.gov/nchs/nhanes.htm).

Table 1 Baseline characteristics, all-cause, and diabetes-related mortality by B2M tertiles. Data are presented as mean \pm S.E.M. for continuous variables and percentage \pm S.E.M. for categorical variables.

\begin{tabular}{|c|c|c|c|c|c|}
\hline & Overall & Tertile 1 & Tertile 2 & Tertile 3 & $P$ value \\
\hline$n$ & 896 & 301 & 296 & 299 & \\
\hline Age (years) & $59.62 \pm 1.06$ & $52.97 \pm 1.17$ & $63.68 \pm 1.41$ & $67.36 \pm 1.75$ & $<0.001$ \\
\hline Women (\%) & $46.8 \pm 4.0$ & $39.5 \pm 5.8$ & $54.4 \pm 6.4$ & $51.7 \pm 5.6$ & 0.096 \\
\hline \multicolumn{6}{|l|}{ Race/ethnicity } \\
\hline Non-hispanic white (\%) & $71.7 \pm 3.3$ & $69.1 \pm 5.3$ & $73.9 \pm 4.3$ & $73.8 \pm 3.3$ & 0.38 \\
\hline Non-hispanic black (\%) & $15.8 \pm 2.1$ & $16.1 \pm 2.9$ & $15.8 \pm 3.1$ & $15.4 \pm 2.2$ & 0.82 \\
\hline Mexican-American (\%) & $6.3 \pm 1.0$ & $8.9 \pm 2.1$ & $4.2 \pm 1.0$ & $4.0 \pm 0.8$ & 0.006 \\
\hline Other (\%) & $6.2 \pm 1.7$ & $5.9 \pm 2.6$ & $6.1 \pm 2.0$ & $6.8 \pm 2.9$ & 0.797 \\
\hline BMI $\left(\mathrm{kg} / \mathrm{m}^{2}\right)$ & $30.62 \pm 0.43$ & $30.68 \pm 0.70$ & $30.90 \pm 0.84$ & $30.16 \pm 0.52$ & 0.651 \\
\hline \multicolumn{6}{|l|}{ Education } \\
\hline Less than the high-school level (\%) & $13.1 \pm 1.6$ & $10.7 \pm 2.1$ & $14.6 \pm 2.0$ & $15.8 \pm 3.0$ & 0.171 \\
\hline High-school level (\%) & $30.0 \pm 3.4$ & $31.3 \pm 6.5$ & $23.9 \pm 3.3$ & $34.6 \pm 3.4$ & 0.824 \\
\hline Greater than the high-school level (\%) & $56.9 \pm 3.2$ & $58.0 \pm 6.2$ & $61.5 \pm 4.1$ & $49.6 \pm 4.0$ & 0.365 \\
\hline Physically active (\%) & $32.0 \pm 3.0$ & $27.7 \pm 4.8$ & $33.4 \pm 4.5$ & $38.3 \pm 4.3$ & 0.134 \\
\hline \multicolumn{6}{|l|}{ Smoking } \\
\hline Never (\%) & $38.9 \pm 3.2$ & $32.8 \pm 5.3$ & $43.7 \pm 6.1$ & $44.8 \pm 4.6$ & 0.108 \\
\hline Former (\%) & $40.1 \pm 4.1$ & $41.6 \pm 7.3$ & $41.5 \pm 6.3$ & $35.9 \pm 4.3$ & 0.582 \\
\hline Current (\%) & $20.9 \pm 2.0$ & $25.6 \pm 5.2$ & $14.8 \pm 3.5$ & $19.3 \pm 2.7$ & 0.245 \\
\hline Microalbuminuria (\%) & $33.2 \pm 3.5$ & $21.9 \pm 5.3$ & $42.2 \pm 5.2$ & $43.7 \pm 5.7$ & 0.007 \\
\hline Serum cholesterol (mg/dl) & $223.63 \pm 2.81$ & $221.03 \pm 4.46$ & $229.07 \pm 3.74$ & $222.15 \pm 5.52$ & 0.74 \\
\hline Serum C-reactive protein (mg/dl) & $0.71 \pm 0.09$ & $0.57 \pm 0.12$ & $0.71 \pm 0.10$ & $0.99 \pm 0.12$ & 0.046 \\
\hline Serum uric acid (mg/dl) & $5.79 \pm 0.14$ & $5.28 \pm 0.25$ & $5.90 \pm 0.19$ & $6.63 \pm 0.14$ & $<0.001$ \\
\hline Serum blood urea nitrogen (mg/dl) & $16.81 \pm 0.37$ & $14.67 \pm 0.60$ & $15.79 \pm 0.28$ & $22.00 \pm 1.17$ & $<0.001$ \\
\hline Serum vitamin D (ng/ml) & $26.29 \pm 0.64$ & $27.23 \pm 1.17$ & $26.23 \pm 0.75$ & $24.60 \pm 0.76$ & 0.074 \\
\hline Hemoglobin (g/dl) & $14.40 \pm 0.08$ & $14.79 \pm 0.10$ & $14.28 \pm 0.10$ & $13.82 \pm 0.14$ & $<0.001$ \\
\hline eGFR $\left(\mathrm{ml} / \mathrm{min} / 1.73 \mathrm{~m}^{2}\right)$ & $67.09 \pm 1.50$ & $77.29 \pm 1.83$ & $63.94 \pm 1.36$ & $51.65 \pm 2.72$ & $<0.001$ \\
\hline Systolic blood pressure $(\mathrm{mm} / \mathrm{Hg})$ & $136.40 \pm 1.21$ & $130.56 \pm 1.60$ & $142.93 \pm 1.66$ & $139.73 \pm 1.94$ & $<0.001$ \\
\hline HDL cholesterol (mg/dl) & $44.41 \pm 1.02$ & $43.43 \pm 1.57$ & $44.59 \pm 1.49$ & $46.06 \pm 1.34$ & 0.263 \\
\hline Serum triglycerides (mg/dl) & $227.69 \pm 10.13$ & $222.12 \pm 11.27$ & $226.67 \pm 21.04$ & $239.36 \pm 15.72$ & 0.398 \\
\hline White blood cell count & $7.75 \pm 0.18$ & $7.65 \pm 0.26$ & $7.94 \pm 0.35$ & $7.73 \pm 0.22$ & 0.672 \\
\hline$\beta-2$ Microglobulin (mg/l) & $2.33 \pm 0.06$ & $1.64 \pm 0.03$ & $2.30 \pm 0.02$ & $3.64 \pm 0.09$ & $<0.001$ \\
\hline Diabetes mortality (\%) & $16.8 \pm 2.0$ & $9.9 \pm 2.9$ & $12.0 \pm 1.7$ & $35.5 \pm 5.2$ & $<0.001$ \\
\hline All-cause mortality (\%) & $42.4 \pm 3.6$ & $21.2 \pm 4.5$ & $43.3 \pm 4.6$ & $81.2 \pm 4.8$ & $<0.001$ \\
\hline
\end{tabular}




\section{B2M measurement}

Serum B2M was measured using the N Latex B2M assay (Siemens Diagnostics, IL, USA). The assay range of B2M was $0.7-23 \mathrm{mg} / \mathrm{l}$. The inter-assay coefficient of variation was $2.7 \%$ (mean $1.757 \mathrm{mg} / \mathrm{l}$ ).

\section{Mortality follow-up}

The association of B2M with mortality was evaluated for deaths from all causes and diabetes-related causes. The NHANES III linked-mortality file was created by matching NHANES III with national death index (NDI) death certificate records (12). As deaths usually have more than one cause, in addition to the reported only one underlying cause of death, NDI records also reports up to 20 contributing causes of death. The data on diabetes as the cause of death were created by the National Center for Health Statistics and included in the NHANES mortality data file. Diabetes was defined as the cause of death if ICD-9 code '250' (representing diabetes mellitus) or ICD-10 codes 'E10-E14' (representing insulin-dependent diabetes mellitus, non-insulin-dependent diabetes mellitus, malnutrition-related diabetes mellitus, other specified diabetes mellitus, and unspecified diabetes mellitus respectively) were coded in any one of the 20 contributing or multiple causes of death fields in the NDI record. The decimals in the codes refer to the complication of diabetes mellitus. Detailed definitions are provided in ICD-9 (http://www.icd9data. com) and ICD-10 (http://www.icd10data.com) websites. As diabetes is rarely the sole cause of death, 'diabetes as the cause of death' coded as 'yes' indicates that it is one of the contributing causes of death. Among those deaths for which diabetes was included as one of the multiple causes of death, a large proportion (39\%) of them were reported to have CVD as an underlying cause of death (13). Length of follow-up for each participant was calculated as the time from the NHANES III examination date to the end of follow-up (date of death or December 31, 2006).

\section{Statistical analyses}

In primary analysis, serum B2M was modeled as a continuous variable (expressed as one natural-log unit increase in serum B2M), tertile (T1, T2, and $\mathrm{T} 3$ ), and threshold model (T1-T2 vs T3). The cutoff values of the tertiles were $<1.99,1.99-2.68$, and $>2.68$. Using the lowest tertile as reference, the hazard ratio (HR) and 95\% CI for each tertile were calculated using univariable and multivariable Cox regression models. To gain additional insight into the potential nonlinearity of the effect of B2M, we examined the Cox regression models using penalized splines. The proportional hazards assumption was evaluated by including time-dependent covariates in the model and found that smoking violated the proportional hazard assumption. Smoking was subsequently included as a stratum in the Cox regression model. A $P$ value of $\leq 0.05$ was considered statistically significant. Variables with skewed distribution were natural log-transformed before analysis. We also performed sensitivity analysis to investigate the association between serum B2M and mortality after exclusion of participants who died within the first 2 years of follow-up.

Sample weights that account for the unequal probabilities of selection, oversampling, and nonresponse were applied in all analyses using the complex sampling module in SPSS version 18.0 Software (SPSS, Inc., Chicago, IL, USA) or R version 2.15.0 (www.R-project.org). All values presented are weighted to represent the USA civilian population.

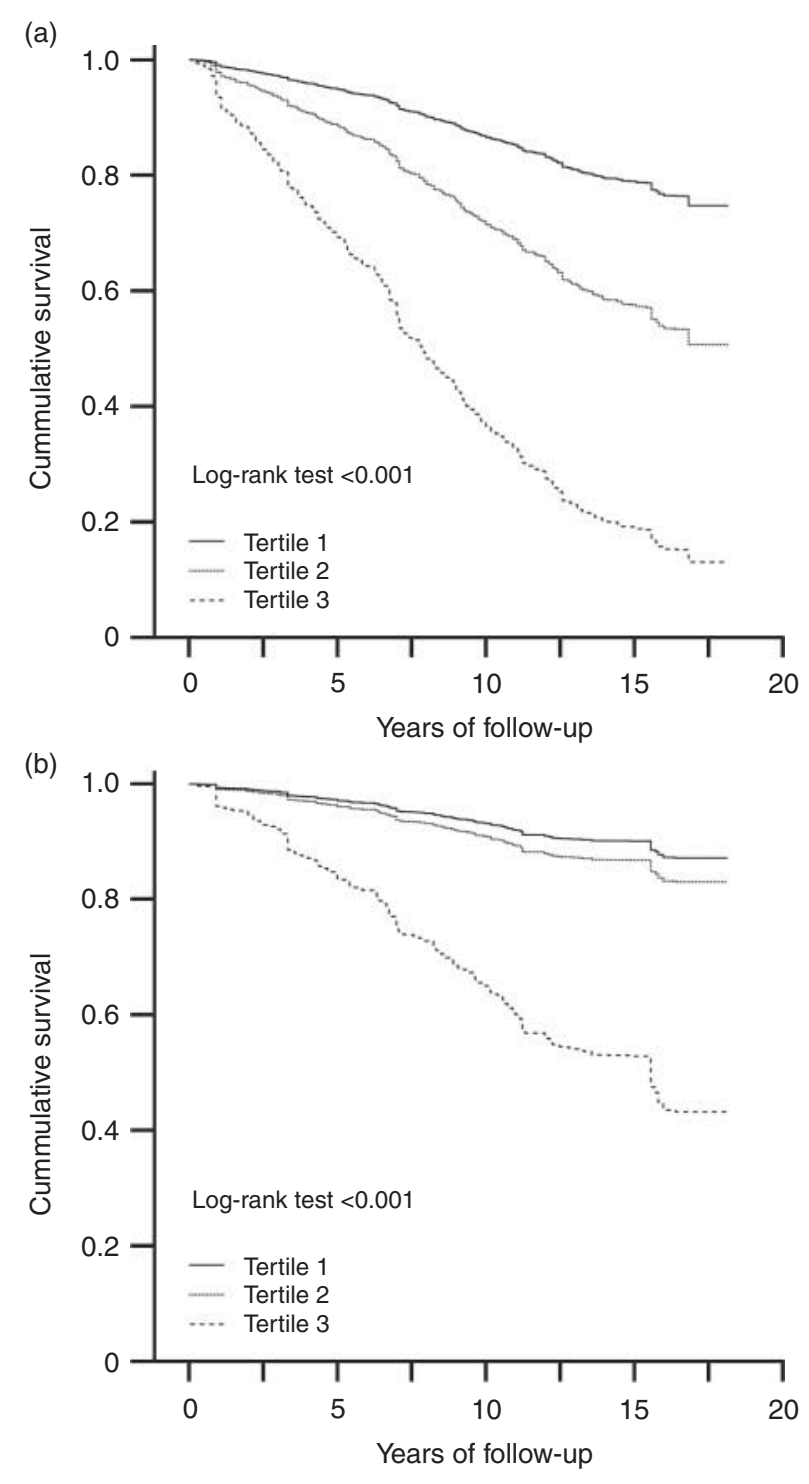

Figure 1 Kaplan-Meier cumulative survival curves for (a) all-cause and (b) diabetes-related mortality depending on tertiles of B2M. 


\section{Results}

\section{Baseline characteristics}

During a median follow-up of 11.8 years (range 0.1-18.2 years) and 9220.5 person/years, 541 $(42.4 \%)$ and $207(16.8 \%)$ participants died from all causes and diabetes-related causes respectively. Detailed cause of death with percentage $\geq 1 \%$ is provided in Supplementary Table 1, see section on supplementary data given at the end of this article. At baseline, the mean age was 59.6 years and $46.8 \%$ of participants were women (Table 1). Participants with higher B2M levels were: older; less likely to be Mexican-American; more likely to have microalbuminuria; more likely to have higher levels of CRP, uric acid, BUN, and systolic blood pressure; and more likely to have lower levels of hemoglobin and eGFR (Table 1). Kaplan-Meier survival curves for all-cause and diabetes-related mortality stratified by B2M tertiles are shown in Fig. 1. Participants with higher levels of $\mathrm{B} 2 \mathrm{M}$ are more likely to die from all causes and diabetes-related causes.

\section{Cox regression models}

The results from Cox regression analysis are shown in Table 2. In model 1, one natural-log unit of $\mathrm{B} 2 \mathrm{M}$ was associated with all-cause mortality with a $\mathrm{HR}=6.53$ (95\% CI 2.07-20.6). Further adjustment attenuated the association, but it remained statistically significant. In the categorical model and threshold model with full adjustment, tertile 3 was associated with all-cause mortality with a $\mathrm{HR}=3.18(95 \%$ CI $1.40-7.20)$ and 2.89 (95\% CI 1.73-4.81) when compared with tertile 1 and tertiles 1-2 respectively. Examination of regression splines suggests a linear increase in hazard for all-cause mortality with increasing B2M levels (Fig. 2).

For diabetes mortality, one natural-log unit of B2M was associated with diabetes-related mortality with a $\mathrm{HR}=8.4(95 \%$ CI 4.31-16.38) in model 1. Further adjustment attenuated the association, but it remained statistically significant. In the categorical model, tertile 3 was associated with diabetes-related mortality in models 1 and 2, and the association became marginally significant $(\mathrm{HR}=3.43,95 \% \mathrm{CI} 0.91-13.03, P=0.069)$ upon further adjustment in model 3. However, tertile 3 remained significantly associated with diabetes-related mortality in the threshold model, regardless of covariate adjustment (Table 2, models 1-3; $P<0.01$ ). Examination of regression splines suggests a linear increase in hazard for diabetes-related mortality with increasing B2M levels (Fig. 2).

Table 2 Hazard ratio (HR) of all-cause and diabetes-related mortality by quartiles of B2M. Data are presented as $\mathrm{HR}(95 \% \mathrm{Cl})$.

\begin{tabular}{|c|c|c|c|c|c|}
\hline & \multirow{2}{*}{$\begin{array}{l}\text { B2M cut points } \\
(\mathrm{mg} / \mathrm{l})\end{array}$} & \multicolumn{2}{|c|}{ All-cause mortality } & \multicolumn{2}{|c|}{ Diabetes mortality } \\
\hline & & $\mathrm{HR}(95 \% \mathrm{Cl})$ & $P$ value & $\mathrm{HR}(95 \% \mathrm{Cl})$ & $P$ value \\
\hline \multicolumn{6}{|c|}{ Per 1 natural-log unit increase of B2M } \\
\hline Model 1 & & $8.49(5.31-13.57)$ & $<0.001$ & $8.4(4.31-16.38)$ & $<0.001$ \\
\hline Model 2 & & $3.74(2.25-6.22)$ & $<0.001$ & $5.81(2.64-12.78)$ & $<0.001$ \\
\hline Model 3 & & $6.53(2.07-20.60)$ & 0.002 & $7.35(1.01-53.38)$ & 0.049 \\
\hline \multicolumn{6}{|c|}{ Categorical models } \\
\hline \multicolumn{6}{|c|}{ Model 1} \\
\hline Tertile 1 & $<1.99$ & 1 (Reference) & & 1 (Reference) & \\
\hline Tertile 2 & $1.99-2.68$ & $2.33(1.31-4.15)$ & 0.005 & $1.42(0.70-2.87)$ & 0.323 \\
\hline Tertile 3 & $>2.68$ & $7.12(3.89-13.06)$ & $<0.001$ & $6.37(2.66-15.28)$ & $<0.001$ \\
\hline \multicolumn{6}{|l|}{ Model 2} \\
\hline Tertile 1 & $<1.99$ & 1 (Reference) & & 1 (Reference) & \\
\hline Tertile 2 & $1.99-2.68$ & $1.23(0.76-2.00)$ & 0.398 & $0.95(0.46-1.97)$ & 0.889 \\
\hline Tertile 3 & $>2.68$ & $3.01(1.60-5.69)$ & 0.001 & $3.95(1.42-10.99)$ & 0.01 \\
\hline \multicolumn{6}{|l|}{ Model 3} \\
\hline Tertile 1 & $<1.99$ & 1 (Reference) & & 1 (Reference) & \\
\hline Tertile 2 & $1.99-2.68$ & $1.15(0.62-2.14)$ & 0.656 & $0.91(0.38-2.15)$ & 0.817 \\
\hline Tertile 3 & $>2.68$ & $3.18(1.40-7.20)$ & 0.007 & $3.43(0.91-13.03)$ & 0.069 \\
\hline \multicolumn{6}{|c|}{ Threshold models } \\
\hline \multicolumn{6}{|c|}{ Model 1} \\
\hline Tertiles 1-2 & $\leq 2.68$ & 1 (Reference) & $<0.001$ & 1 (Reference) & $<0.001$ \\
\hline Tertile 3 & $>2.68$ & $4.83(3.09-7.53)$ & & $5.57(2.79-11.12)$ & \\
\hline \multicolumn{6}{|l|}{ Model 2} \\
\hline Tertiles 1-2 & $\leq 2.68$ & 1 (Reference) & $<0.001$ & 1 (Reference) & $<0.001$ \\
\hline Tertile 3 & $>2.68$ & $2.65(1.70-4.15)$ & & $4.05(1.88-8.72)$ & \\
\hline \multicolumn{6}{|l|}{ Model 3} \\
\hline Tertiles 1-2 & $\leq 2.68$ & 1 (Reference) & $<0.001$ & 1 (Reference) & 0.009 \\
\hline Tertile 3 & $>2.68$ & $2.89(1.73-4.81)$ & & $3.63(1.40-9.43)$ & \\
\hline
\end{tabular}

Model 1: unadjusted. Model 2: adjusted for lifestyle factors (age, education, physical activity, race/ethnicity, and sex). Model 3: further adjusted for BMI, serum total cholesterol, HDL cholesterol, triglycerides, eGFR, systolic blood pressure, total white blood cell count, hemoglobin, CRP, and microalbuminuria. B2M, $\beta-2$ microglobulin. 


\section{Discussion}

In this study, we have shown that serum B2M level is a powerful predictor of mortality in people with diabetes, and the effect may be independent of renal function. Since tools for predicting overall and cause-specific mortality in diabetic patients are limited, our study provides new insights into the role of B2M in mortality and suggests that it may be a useful prognostic marker in diabetes.

In the current study, we showed that B2M is a predictor of both all-cause and diabetes-related mortality. It is clear that the association was strongly dependent on lifestyle factors, while further adjustment of cardiometabolic risk factors did not attenuate the associations much. In general, the effect of B2M on diabetes mortality was slightly stronger than that of all-cause mortality (Table 2), and this observation might be due to the fact that higher serum B2M levels are associated with multiple diabetic complications, and therefore worsen outcomes.

Diabetic nephropathy is a major complication of diabetes. Previous studies have shown that the indices of renal function are important predictors of mortality in diabetes patients, such as reduced eGFR $(2,14,15)$ and albuminuria $(2,14,15,16)$. However, there are a few novel biomarkers of renal function that may surpass eGFR, such as B2M and cystatin C. In 691 diabetic participants from the Cardiovascular Health Study (CHS), participants having cystatin C-eGFR $<60$ had a $\mathrm{HR}=1.73(95 \%$ CI $1.37-2.18)$ and 1.71 (95\% CI 1.21-2.42) for all-cause and cardiovascular mortality respectively, which are higher than the HRs estimated using the Modification of Diet in Renal Disease (MDRD)eGFR (2). Subsequent analysis also showed that cystatin $\mathrm{C}$-eGFR added extra predictive value, in terms of C-statistics, to MDRD-eGFR (2). Similarly, the association between B2M and all-cause and diabetes-related mortality remained robust even after adjustment of eGFR. Altogether, these observations suggest that the effect of B2M on mortality may be independent of renal function, and may have an additional role in contributing to the risk of mortality.

B2M has multiple biological functions. B2M is a part of the major histocompatibility complex (MHC) class 1 complex and an initiator of inflammatory response (17) that triggers the inflammatory process (18). For example, B2M can induce the expression of cytokines, adhesion molecules, and metalloproteinase, such as vascular cell adhesion molecule 1 , metalloproteinase 1 , and cyclooxygenase 2 , through changes in inositol 1,4,5-trisphosphate-mediated intracellular calcium, actin cytoskeleton rearrangement, and phosphatidylinositol-3 kinase (17). Several inflammatory cytokines, such as IL1 and TNF $\alpha$, can also increase the expression of MHC class 1 and thereby that of B2M $(19,20)$. Serum $\mathrm{B} 2 \mathrm{M}$ level is increased in several autoimmune diseases, such as systemic lupus erythematosus (21) and (a)

All-cause death

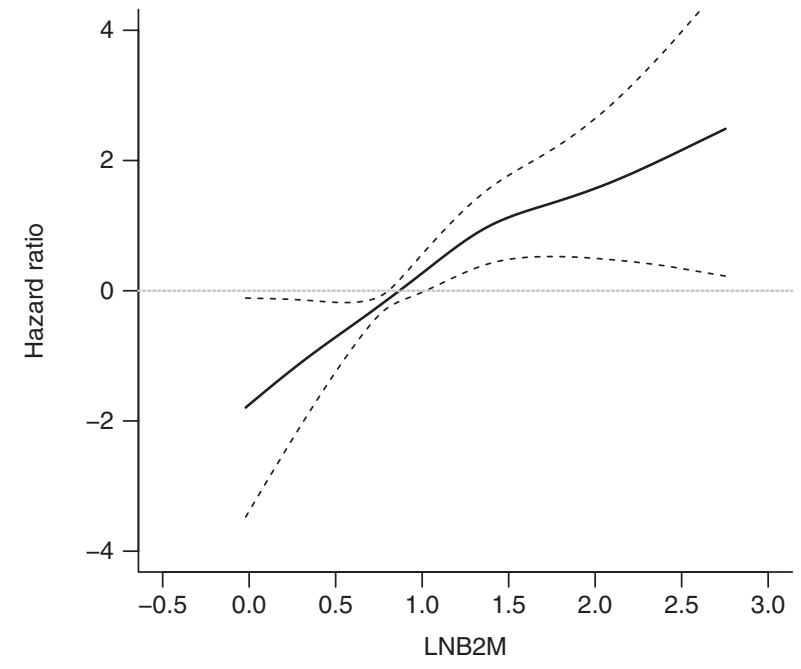

(b)

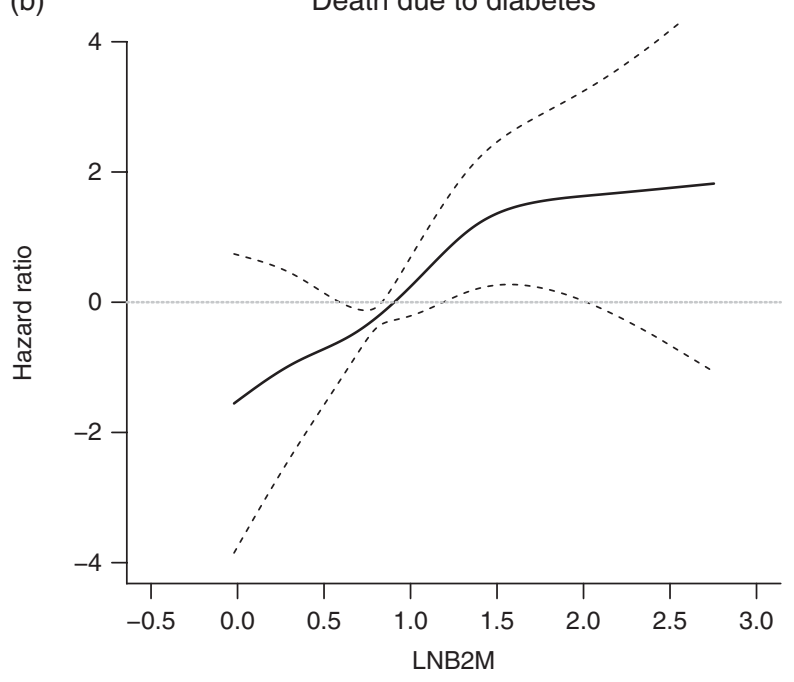

Figure 2 Association between serum $\beta-2$ microglobulin and mortality assessed via penalized regression splines. (a) All-cause mortality and (b) diabetes-related mortality.

rheumatoid arthritis (22). In the current study, we observed that inclusion of CRP slightly attenuated the association of B2M (Supplementary Table 2, see section on supplementary data given at the end of this article), indicating that the association was partly mediated by inflammation. B2M is amyloidogenic, which leads to amyloidosis when serum B2M level is high (23). Moreover, hyperglycemia leads to the formation of AGEs, wherein AGEs of B2M, such as ribosylated $\mathrm{B} 2 \mathrm{M}$, is cytotoxic because of the generation of reactive oxygen species (8). Future studies are required to elucidate the role of glycated B2M as well as AGE modification of B2M in diabetes.

Our study has several strengths. The study population is large, multiethnic, nationally representative, 
and well-characterized with data on multiple risk factors and confounders. The long follow-up and the large number of events provided ample statistical power. Meaningful gains in clinical utility were demonstrated. For example, the HR of B2M with mortality is $>2$, which is considered to be clinically significant. Nevertheless, there are limitations. First, only a single baseline measurement of $\mathrm{B} 2 \mathrm{M}$ and fasting glucose was performed; repeated measurements may provide more clues on whether high B2M and fasting glucose have a synergistic effect on mortality. Our results showing the predictive power of a single baseline measurement of $\mathrm{B} 2 \mathrm{M}$ are therefore all the more remarkable. Second, $\mathrm{B} 2 \mathrm{M}$ is an indispensible prognostic marker of several cancers, while we also observed that B2M is a predictor of cancer-related mortality in the current study. Compared with tertile 1 , the HRs of tertiles 2 and 3 were $1.49(0.71-3.16)$ and $2.71(1.11-6.60)$ respectively (trend $P=0.031$ ). Thus, the association with mortality may be driven by participants with incipient cancer. However, in our sensitivity analysis excluding participants who died within the first 2 years of followup, B2M remained significantly associated with mortality (Supplementary Table 3, see section on supplementary data given at the end of this article). Third, our findings may not be generalizable to other populations. Future prospective studies in other populations are warranted. Our results have opened up the possibility that measures that reduce B2M may also reduce mortality. For example, anti-B2M antibody is currently under development as an intervention to treat cancer (24). A previous study showed that it induces apoptosis of myeloma cells but spares normal cells. However, long-term clinical trials are needed to test whether interventions that reduce serum B2M can improve survival in diabetic patients. Fourth, the adjustment model in the current study may not be adequate to examine whether the effect of $\mathrm{B} 2 \mathrm{M}$ on mortality is totally independent of renal function and inflammation. In model 3, we could only include one of the highly correlated risk factors, such as total cholesterol and LDL cholesterol. Lastly, the current study defined diabetes-associated mortality based on ICD codes listed in NDI; the actual cause of death could not be determined with certainty.

In conclusion, serum B2M levels predict mortality for people with diabetes. Clinical studies are needed to verify whether interventions that lower B2M would also lower mortality.

\section{Supplementary data}

This is linked to the online version of the paper at http://dx.doi.org/10. 1530/EJE-13-0003.

\section{Declaration of interest}

The authors declare that there is no conflict of interest that could be perceived as prejudicing the impartiality of the research reported.

\section{Funding}

B M Y Cheung receives support from the Sun Chieh Yeh Heart Foundation. C-L Cheung is supported by the Faculty Development Fund, Li Ka Shing Faculty of Medicine, University of Hong Kong.

\section{References}

1 Weiner DE, Tighiouart H, Amin MG, Stark PC, MacLeod B, Griffith JL, Salem DN, Levey AS \& Sarnak MJ. Chronic kidney disease as a risk factor for cardiovascular disease and allcause mortality: a pooled analysis of community-based studies. Journal of the American Society of Nephrology 200415 1307-1315. (doi:10.1097/01.ASN.0000123691.46138.E2)

2 de Boer IH, Katz R, Cao JJ, Fried LF, Kestenbaum B, Mukamal K, Rifkin DE, Sarnak MJ, Shlipak MG \& Siscovick DS. Cystatin C, albuminuria, and mortality among older adults with diabetes. Diabetes Care 200932 1833-1838. (doi:10.2337/dc09-0191)

3 Liabeuf S, Lenglet A, Desjardins L, Neirynck N, Glorieux G, Lemke HD, Vanholder R, Diouf M, Choukroun G \& Massy ZA. Plasma $\beta$-2 microglobulin is associated with cardiovascular disease in uremic patients. Kidney International 201282 1297-1303. (doi:10.1038/ki.2012.301)

4 Astor BC, Shafi T, Hoogeveen RC, Matsushita K, Ballantyne CM, Inker LA \& Coresh J. Novel markers of kidney function as predictors of ESRD, cardiovascular disease, and mortality in the general population. American Journal of Kidney Diseases 201259 653-662. (doi:10.1053/j.ajkd.2011.11.042)

5 Cheung AK, Greene T, Leypoldt JK, Yan G, Allon M, Delmez J, Levey AS, Levin NW, Rocco MV, Schulman G et al. Association between serum 2-microglobulin level and infectious mortality in hemodialysis patients. Clinical Journal of the American Society of Nephrology 20083 69-77. (doi:10.2215/CJN.02340607)

6 Shinkai S, Chaves PH, Fujiwara Y, Watanabe S, Shibata H, Yoshida H \& Suzuki T. $\beta 2$-Microglobulin for risk stratification of total mortality in the elderly population: comparison with cystatin $\mathrm{C}$ and C-reactive protein. Archives of Internal Medicine 2008168 200-206. (doi:10.1001/archinternmed.2007.64)

7 Lonnemann G \& Koch KM. $\beta(2)$-Microglobulin amyloidosis: effects of ultrapure dialysate and type of dialyzer membrane. Journal of the American Society of Nephrology 200213 (Suppl 1) S72-S77.

8 Kong FL, Cheng W, Chen J \& Liang Y. D-Ribose glycates $\beta(2)$-microglobulin to form aggregates with high cytotoxicity through a ROS-mediated pathway. Chemico-Biological Interactions 2011194 69-78. (doi:10.1016/j.cbi.2011.08.003)

9 Freeman WM, Bixler GV, Brucklacher RM, Walsh E, Kimball SR, Jefferson LS \& Bronson SK. Transcriptomic comparison of the retina in two mouse models of diabetes. Journal of Ocular Biology, Diseases, and Informatics 20092 202-213. (doi:10.1007/s12177009-9045-3)

10 Centers for Disease Control and Prevention (CDC). National Center for Health Statistics (NCHS). National Health and Nutrition Examination Survey Data. Hyattsville, MD, USA. Department of Health and Human Services, Centers for Disease Control and Prevention (1988-1994), 1996.

11 Levey AS, Stevens LA, Schmid CH, Zhang YL, Castro AF III, Feldman HI, Kusek JW, Eggers P, Van Lente F, Greene T et al. A new equation to estimate glomerular filtration rate. Annals of Internal Medicine 2009150 604-612. (doi:10.7326/0003-4819-150-9200905050-00006)

12 National Center for Health Statistics. Office of Analysis and Epidemiology, The Third National Health and Nutrition Examination Survey (NHANES III) Linked Mortality File, Mortality follow-up through 2006: Matching Methodology May 2009. Hyattsville, Maryland. (Available at the following address: http:// www.cdc.gov/nchs/data/datalinkage/matching_methodology_ nhanes3_final.pdf), 2009. 
13 Murray CJ, Dias RH, Kulkarni SC, Lozano R, Stevens GA \& Ezzati M. Improving the comparability of diabetes mortality statistics in the U.S. and Mexico. Diabetes Care 200831 451-458. (doi:10.2337/dc07-1370)

14 Berhane AM, Weil EJ, Knowler WC, Nelson RG \& Hanson RL. Albuminuria and estimated glomerular filtration rate as predictors of diabetic end-stage renal disease and death. Clinical Journal of the American Society of Nephrology 20116 2444-2451. (doi:10.2215/ CJN.00580111)

15 Drury PL, Ting R, Zannino D, Ehnholm C, Flack J, Whiting M, Fassett R, Ansquer JC, Dixon P, Davis TM et al. Estimated glomerular filtration rate and albuminuria are independent predictors of cardiovascular events and death in type 2 diabetes mellitus: the Fenofibrate Intervention and Event Lowering in Diabetes (FIELD) Study. Diabetologia 201154 32-43. (doi:10.1007/s00125-010-1854-1)

16 Bruno G, Merletti F, Bargero G, Novelli G, Melis D, Soddu A, Perotto M, Pagano G \& Cavallo-Perin P. Estimated glomerular filtration rate, albuminuria and mortality in type 2 diabetes: the Casale Monferrato Study. Diabetologia 200750 941-948. (doi:10.1007/s00125-007-0616-1)

17 Xie J \& Yi Q. $\beta 2-$ Microglobulin as a potential initiator of inflammatory responses. Trends in Immunology $2003 \mathbf{2 4}$ 228-229 (author reply 229-230). (doi:10.1016/S14714906(03)00076-0)

18 Chen NX, O’Neill KD, Niwa T \& Moe SM. Signal transduction of $\beta 2 \mathrm{~m}$-induced expression of VCAM-1 and COX-2 in synovial fibroblasts. Kidney International $2002 \mathbf{6 1} 414-424$. (doi:10.1046/j.1523-1755.2002.00136.x)
19 Nachbaur K, Troppmair J, Bieling P, Kotlan B, Konig P \& Huber C. Cytokines in the control of $\beta-2$ microglobulin release. I. In vitro studies on various haemopoietic cells. Immunobiology 1988177 55-65. (doi:10.1016/S0171-2985(88)80091-3)

20 Fuchs D, Hausen A, Reibnegger G, Werner ER, Felmayer GW \& Wachter H. $\beta 2-$ Microglobulin and immune activation. Clinical Chemistry 198935 2158-2159.

21 Kim HA, Jeon JY, Yoon JM \& Suh CH. $\beta 2$-Microglobulin can be a disease activity marker in systemic lupus erythematosus. American Journal of the Medical Sciences 2010339 337-340. (doi:10.1097/ MAJ.Ob013e3181d26dfb)

22 Manicourt D, Brauman H \& Orloff S. Plasma and urinary levels of $\beta 2$ microglobulin in rheumatoid arthritis. Annals of the Rheumatic Diseases 197837 328-332. (doi:10.1136/ard.37.4.328)

23 Sonikian M, Gogusev J, Zingraff J, Loric S, Quednau B, Bessou G, Siffert W, Drueke TB, Reusch HP \& Luft FC. Potential effect of metabolic acidosis on $\beta 2$-microglobulin generation: in vivo and in vitro studies. Journal of the American Society of Nephrology 19967 350-356.

24 Yang J \& Yi Q. Killing tumor cells through their surface $\beta(2)$-microglobulin or major histocompatibility complex class I molecules. Cancer 2010116 1638-1645. (doi:10.1002/cncr. 24953)

Received 3 January 2013

Revised version received 4 April 2013

Accepted 9 April 2013 